\title{
Cladistics
}

Cladistics 20 (2004) 23-31

www.blackwell-synergy.com

\section{Transformation Series as an Ideographic Character Concept}

\author{
Taran Grant ${ }^{\mathrm{a}, *}$ and Arnold G. Kluge \\ ${ }^{a}$ Division of Vertebrate Zoology, Herpetology, American Museum of Natural History, New York, NY 10024, USA; Department of Ecology, Evolution, \\ and Environmental Biology, Columbia University, New York, NY 10027, USA \\ ${ }^{b}$ Museum of Zoology, University of Michigan, Ann Arbor, MI 48109, USA \\ Accepted 23 December 2003
}

\begin{abstract}
An ideographic concept of character is indispensable to phylogenetic inference. Hennig proposed that characters be conceptualized as "transformation series", a proposal that is firmly grounded in evolutionary theory and consistent with the method of inferring transformation events as evidence of phylogenetic propinquity. Nevertheless, that concept is usually overlooked or rejected in favor of others based on similarity. Here we explicate Hennig's definition of character as an ideographic concept in the science of phylogenetic systematics. As transformation series, characters are historical individuals akin to species and clades. As such, the related concept of homology refers to a historical identity relation and is not equivalent to or synonymous with synapomorphy. The distinction between primary and secondary homology is dismissed on the grounds that it conflates the concept of homology with the discovery operations used to detect instances of that concept. Although concern for character dependence is generally valid, it is often misplaced, focusing on functional or developmental correlation (both of which are irrelevant in phylogenetic systematics but may be valid in other fields) instead of the historical/transformational independence relevant to phylogenetic inference. As an ideographic science concerned with concrete objects and events (i.e. individuals), intensionally and extensionally defined properties are inconsistent with the individuation of characters for phylogenetic analysis, the utility of properties being limited to communicating results and facilitating future rounds of testing.
\end{abstract}

(C) The Willi Hennig Society 2004.

\section{Introduction}

Ordinary discourse is made difficult by the many meanings of the word character, as seen in the Oxford English Dictionary. The difficulty is no less in particular fields of science. For example, Colless's (1985, p. 230) survey of over 50 books and articles revealed the following explicitly stated, or implied, meanings of character in systematics: "an attribute, a set of attributes, a feature, a characteristic, a property, a part, a morphocline, a differentia, an homology, a truth, a theory, an aspect (of an organism), a basis for comparison, a similarity, and (mathematically) a variable, a function, a mapping, an equivalence relation, and a set of probability distributions." Colless (p. 231) even

\footnotetext{
*Corresponding author. Division of Vertebrate Zoology, Herpetology, American Museum of Natural History, New York, NY 10024, USA.

E-mail address: grant@amnh.org
}

added Hennig's (1966) definition of character to his list, as "transformation series", interpreting Hennig to have meant "a set of mutually exclusive 'character conditions' or 'special characters"'. Thus it is that many evolutionary biologists, including many phylogeneticists, have failed to understand Hennig's concept of character- "a historical event in the evolution of a feature" (Fristrup, 1992, p. 45), or as Hennig (1966; p. 93; see also preceding text, beginning on p. 88) clarified: ${ }^{1}$

Different characters that are to be regarded as transformation stages of the same original character are generally called homologous. "Transformation" naturally refers to real historical processes of evolution, and not to the possibility of formally deriving characters from one another in the sense of idealistic morphology.

\footnotetext{
${ }^{1}$ Note that Hennig's (1966) usage of "character" as a discrete stage - minimally a singular heritable event - in a transformation series is equivalent to the conventional usage of "character-state". As such, his "transformation series" is equivalent to what is conventionally referred to as "character".
} 
Moreover, many contemporary workers seem entirely unaware of Hennig's character concept. A recent multiauthored book (Wagner, 2001) dedicated to elucidating the concept of character in evolutionary biology overlooked Hennig's transformation series, as did two philosophical papers (Richards, 2002, 2003) that specifically examined character concepts in phylogenetic systematics. Overlooking or misunderstanding this concept of character has resulted in significant confusion, with far-reaching implications for empirical phylogenetic studies. We believe the most significant level of confusion is between a similarity- or property-based concept of character, as per Colless's list, and character as a series of singular historical events (Platnick, 1979). Hennig's definition is clearly grounded in evolutionary theory, whereas the other definitions are not.

In this paper we explicate the concept of character required of a consistent approach to historical evolutionary biology. By this we mean an approach in which there are no apparent negations or contradictions among the concepts and discovery operations that lead to objective knowledge claims. Most of our views can be traced to Hennig (1966), who in turn drew from Woodger $(1929,1952)$, among others, albeit with numerous technical clarifications admitted over the years. Our underlying premise is that concepts matter; they determine which observations are scientifically relevant and how they may be processed in order to make consistent inferences. Only after theoretically defined concepts have been established, such as the concept of character, can valid discovery operations be developed and implemented in empirical research (Grant, 2002).

\section{Transformation}

Evolutionary biology is concerned with heritable change. Darwin's (1859, p. 420) theory of "descent, with modification", specifies transformation from ancestral to derived conditions $\left(\mathrm{a} \rightarrow \mathrm{a}^{\prime} \rightarrow \mathrm{a}^{\prime \prime}\right.$, etc., where $\rightarrow$ is a transformation event that may involve the modification of a complex phenotypic character or the substitution, insertion, or deletion of a nucleotide) as the explanation of all heritable variation among living things; molecular genetics identifies transformations of DNA as the primary source of heritable variation; and those molecular transformations involve interactions governed ultimately by quantum physics (Stamos, 2001). Because all transformation involves the modification of an existing condition, the course of evolution and the diversification of life are, at least to some degree, a function of historical contingency (historical precedence, historicity, phylogenetic conservatism). As Hull (1988, p. 424) observed, "[t]he past constrains the future only to the extent that it has structured the present", and that requires the genetic and developmental integration of the ancestral phenotype. Thus, while history is not predictive of diversity because of its necessarily unique nature, it may nonetheless be understood as constraining the genotype (and phenotype) by having limited the possible transformations.

Many different aspects of heritable transformation may be studied under the rubric of evolutionary biology, and the validity of the various scientific approaches depends on which aspect is the subject of inquiry. For example, research that focuses on the results or effects of transformation events may investigate the universal statistical principles that govern the spread of transformed character-states through populations, which can inform predictive models of population genetics, or may examine the adaptive or deleterious effects of particular transformations in functional terms. The emphasis placed on such studies in the Modern Synthesis has resulted in the familiar textbook definition of evolution as "change in allele frequency". Although that population-level definition is certainly descriptive of an evolutionary process, it is a grossly biased definition, because it overlooks many other fundamental aspects of transformation in evolution. For example, molecular evolutionary studies investigate the mechanisms that underlie different classes of transformations of DNA, such as insertions and deletions, point mutations, and gene rearrangements. In addition, phylogenetic systematics focuses on the history of the transformation events themselves as evidence of phylogenetic relationships, as is evident in the patristic relationship function. As Farris (1967, p. 47) described it, "The overall patristic difference is the sum of the patristic unit character differences. Each patristic unit character difference is the summation of the changes of that character from point to point over the phyletic line between the populations compared." Due in large part to the reductionist zeal of the Modern Synthesis to force all evolutionary biology into a single inferential framework, the distinct epistemological constraints associated with each kind of problem continue to be overlooked by many workers (Siddall and Kluge, 1997).

Phylogenetic systematics stands in contraposition to most areas of evolutionary biology in that it is explicitly and exclusively historical - it is ideographic. It does not predict evolutionary changes into the future (although it may be predictive of past events not yet detected, i.e. retrodictive), and it does not conjecture universal laws - it is not nomothetic. Instead, it seeks to provide ideographic explanations of the heritable variation observed among lineages related through phylogeny (as opposed to ontogeny or tokogeny) by discovering the series of necessarily unique historical transformation events that occurred during their evolution, where phylogenetic hypotheses are chosen on the basis of the number of independent transformation events (patristic distance) required by the competing propositions 
(Farris, 1967, 1970). That is, phylogenetic systematists use historical transformation events as evidence of phylogenetic propinquity.

The transformationist basis of phylogenetic methods contrasts with phenetic approaches where organisms are classified according to some necessarily arbitrary measure of similarity (e.g. Rieppel and Kearney, 2002; see Kluge, 2003b). Failure to recognize the fundamental distinction between transformation and similarity as the basis for delimiting groups has led some phylogeneticists to mistakenly use phenetic methods to infer phylogeny (Farris, 1967). This is obvious in the case of explicitly similarity-based (albeit weighted) distance methods like minimum evolution and neighbor joining, but it is also true of other methods. For example, three-taxon analysis is based on similarity (Kluge and Farris, 1999), as is the derivative method RASA (Faivovich, 2002; Farris, 2002). Likewise, methods that convert polymorphism into separate, "polymorphic" states or frequencies allow populations to be grouped on the basis of more precise descriptions of similarity, but they mistakenly equate population-level similarity with transformation events (Grant and Kluge, 2003). Only the transformation from one character-state to another, $a \rightarrow a^{\prime}$, constitutes evidence in phylogenetic inference; changes in frequency (including extinction) of states in populations do not entail additional character-state transformations and are therefore evidentially irrelevant. Two populations characterized as a $a^{\prime}$ are certainly more similar to each other than they are to uniformly a or a' populations, but they are not delimited as a distinct group by any transformation event, since the only transformation was from a to $a^{\prime}$. In summary, the fact that phylogenetic inferences are based on transformation events determines the kinds of observations that constitute evidence of relationship. ${ }^{2}$

\section{Characters as historical individuals}

Although transformation events constitute the relevant units of evolutionary change in phylogenetics, there must be some "thing" in existence to undergo transformation. Lineages are usually understood as being

\footnotetext{
${ }^{2}$ Other kinds of data have been proposed as evidence of phylogenetic relationships, such as relative age of origin or stratigraphic position. However, for there to be a direct evidential connection between observed variation of any kind and lineage diversification there must be heritability, and that is not a characteristic of stratigraphic data. Furthermore, the truism that ancestors precede their descendents is only sufficient to establish a linear temporal order, whereas transformation series are inclusively hierarchic. For additional issues in this debate see Fisher (1994), Fox et al. (1999), Heyning et al. (1999), Vermeij (1999, 2001), Smith (2000), Bodenbender and Fisher (2001), Brochu et al. (2001), Geiger et al. (2001), Novacek (2001), Sumrall and Brochu (2003), and Fisher and Bodenbender (2003).
}

composed ultimately of individual organisms (see Frost and Kluge, 1994). Although transformations are necessarily manifested physically as organisms (sensu lato; e.g. Wilson and Sober, 1994), the organism is not the basic unit of evolutionary transformation. Instead, the individual parts of organisms undergo transformation, which justifies decomposing organisms into independently variable character-states to test phylogenetic hypotheses. That an organism transforms by timeordered changes in different parts, rather than by a whole stage in the lifecycle replacing another, is evidence enough to validate the individuation of transformations at the sub-organism level.

This sub-organism focus does not deny the importance of organisms in evolutionary biology and phylogenetic inference: the fact that the elements of evolution are effectively "bundled" into organisms, family groups, demes, populations, and phylogenetic lineages places important constraints on both the way they evolve and the valid inferences that can be made about them. Indeed, it is because they are bundled in this way that the series of transformations of individual parts are attributed evidential significance in the inference of phylogeny (Hennig, 1966).

Character-states have generally been conceptualized as properties (attributes, features), which logically denies their ability to transform or evolve, since properties are class concepts and, as such, are immutable (Kluge, 2003b). Only individuals (in the ontological sense) can undergo change. In the same way that species and clades are conceptualized as increasingly inclusive historical individuals linked by a series of unique diversification events (Kluge, 1990; Grant, 2002), we propose that heritable variations among organisms be conceptualized as increasingly inclusive historical individuals linked by a series of transformation events, i.e. the transformation series of Hennig (1966). Unfortunately, there are those who distort the notion of individuals and classes, so that everything is a class. For example, Boyd (1999) has argued that species are homeostatic cluster kinds; however, in being homeostatic, such kinds cannot evolve (Kluge, 2003a, p. 234).

Since Ghiselin's (1974) "radical solution" (see also Hennig, 1966, pp. 80-83, who argued strongly for the same solution), the biological literature has swelled with discussions of ontological individuality. For the present purposes, two kinds of individuals are distinguished (see Kluge, 1990; Grant, 2002). Contemporary individuals are united by both a common, unique history and cohesive and integrative processes (or, in Hennig's terms, they form a divisional hierarchy and are also operational units). For example, the unity of an organism, such as Darrel Frost, is due to both the historical connections among semaphoronts (e.g. Frost in different stages of his ontogeny) and the cohesive and integrative processes operating presently. In contrast, historical individuals are 
united only by common history, not current processes (i.e. they "lack the character "operational unit"; Hennig, 1966, p. 83). The unity of a monophyletic group, such as Squamata, is exclusively historical; it is neither united by nor involved in cohesive and integrative processes. Just as there are many kinds of contemporary individuals (consider the ontological differences between, say, an individual atom and an individual galaxy), there are many kinds of historical individuals. In phylogenetic systematics, two kinds of historical individuals are relevant: those that are the result of lineage diversification events (and are therefore related by phylogeny), and those that are the result of heritable transformation events. Within this framework, character-states are defined conceptually as the least inclusive historical individuals that result from heritable transformation events.

It is common for character-states to be defined conceptually as purely observational statements, but they are complex, theory-laden hypotheses of historical identity-no less hypothetical than the characters and more inclusive historical individuals of which they are part. What is actually observed are the parts of an individual organism, yet those parts can be individuated according to a variety of criteria, each of which may be relevant to a given problem or in a particular inferential context. Structurally, the basic parts are the fundamental particles of physics (quarks, leptons, photons, gluons, W-bosons, and Z-bosons), although biological problems may treat cells as structurally basic; developmentally, they are the parts united by independent ontogenetic pathways (note that these basic units are also historical individuals); and functionally, they are the operationally independent structures involved in certain processes. That is, independence is contextdependent (e.g. the fact that a single observer scores all terminals renders all entries non-independent in that context), so the context must be specified for relevant independence to be assessed. Because the basic or fundamental unit in phylogenetic inference is the transformation event, the relevant independent parts of organisms are those that have undergone independent, heritable transformation events. What matters in individuating character-states (and more inclusive transformation series), therefore, is not the structural, developmental, or functional independence of a part, but its historical/transformational independence.

That character-states are much more than direct observations is clarified by considering the variation observed among organisms that is subsumed within a given character-state. With the exception of some kinds of molecular characters, for which character-states are precisely delimited in terms of physico-chemical structure, a strict application of the observational characterstate concept would result in a different character-state for every specimen examined, because, as any morphologist can attest (e.g. Hennig, 1966, p. 37), there is always some degree of individual variation. However, given that character-states are conjectured historical individuals, only variation attributable to heritable transformation events is relevant; any variation judged to be non-heritable (i.e. due to environmental factors, not heritable transformations) is defensibly excluded as evidentially irrelevant. Any such claim is empirically testable, and it may be found in future rounds of testing (e.g. through the examination of additional specimens, more precise measurement, direct experimentation) that the original individuation of character-states was inadequate, arbitrary, or otherwise false and must therefore be modified. Fallibility does not equate with subjectivity, however, and the procedure of testing and re-testing character-state individuations is explicitly objective (Kluge, 2003b).

As is true of other kinds of historical individuals, character-states are parts of more inclusive historical individuals linked through time by transformation events, and those more inclusive historical individuals may, in turn, be parts of even more inclusive historical individuals. Stated differently, by definition each historical individual has its own, unique history, so each part of a more inclusive historical individual will have its own, particular history as well, all of which are linked together by necessarily unique transformation events. The dependency between such transformation series is non-problematic, because it merely reflects the transformation event(s) they share, i.e. the shared portion of their history (see below).

In contrast, the dependency that obtains from alternative descriptions of the outcome of the same transformation event represents an obvious problem for phylogenetic inference (Farris, 1983). For example, the DNA sequences TTT, TTC, TTA and the ancestral TTG entail a single independent transformation series corresponding to the variable position in the aligned sequences. TTT and TTC can also be described as the amino acid phenylalanine, and TTA and TTG as leucine. Consistent with the concept of "taxic homology" espoused by Patterson (1982b; not to be confused with the "taxic approach" of Eldredge, 1979; see Farris, 2000; Farris et al., 2001, p. 80) and others, it could be argued that because the hierarchic affinities (relative adjacencies) implied by these "characters" are distinct, they are both valid homology statements that should be combined to simultaneously test phylogenetic hypotheses (Agosti et al., 1996; Freudenstein et al., 2003). However, this reveals the illogic of the concept of "taxic homology", given that it is obvious that these are simply alternative descriptions of the outcome of the same events, regardless of whether they imply the same or different hierarchies. The relationship between TTT and TTC as phenylalanine is one of functional equivalence, not historical identity (homology), and while that may be informative in other areas of inquiry (e.g. studies of 
natural selection or adaptation), it is irrelevant in phylogenetic systematics. Moreover, the different hierarchy (relative adjacency) implied by the amino acid "character" is entirely due to the imprecision imposed by the degeneracy of the genetic code, resulting in a loss of information on transformation events and a false hypothesis of homology. There is a potentially infinite number of ways to describe real entities (such as nucleotides in DNA sequences) and rank them according to similarity (e.g. translated amino acids; number of rings [purine/pyrimidine]; number of atoms [12/14/15]; number of carbon atoms [4/5]; number of nitrogen atoms [2/3/5]; number of hydrogen atoms [4/5]; number of oxygen atoms [0/1/2]; etc.). But this is only a problem for phenetic approaches and their emphasis on similarity and description (e.g. Rieppel and Kearney, 2002), not phylogenetic systematics, which emphasizes transformation and explanation (e.g. Kluge, 2003b).

\section{Homology $=$ historical identity $\neq$ synapomorphy}

Having established the conceptual relationship between transformation events and the things that are transformed, ${ }^{3}$ we can explicate the concepts of homology and synapomorphy. Homology refers to the relation between parts that resulted from the same heritable transformation event. As such, homology = historical identity, i.e. two or more parts that are homologous are parts of the same historical individual (Kluge, 2003a,b). However, recalling that historical individuals may be composed of more and/or less inclusive parts, two or more parts may be homologous in one context but not in another. The particular statement of historical identity must therefore refer to the specific historical individual in question, and, transitively, the relevant transformation event. For example, bird wings and bat wings are homologous as tetrapod fore-appendages (which refers to a single transformation event) but not as wings (which refers to distinct, historically unrelated transformation events). This definition of homology as historical identity provides a theoretical basis for the correspondence of parts of equivalent semaphoronts, parts of the same

\footnotetext{
${ }^{3}$ Of course, this was already clarified by Hennig (1966, p. 89), who cautioned, "we must always be aware of the fact that 'characters' that can be compared are basically only character conditions that the real process of evolution produced by transformation of an original condition." Generalizing further, Woodger (1929, pp. 301-302) had already pointed out that, because space and time are coextensive, so too are the event and the thing: "the perceptual object we also call the organism is expressive of certain of the knowable characteristics of the event which can be exemplified in sense-experience" (i.e. the organism is the event and the event is the organism). Although Woodger phrased his discussion in terms of the organism, the relation extends transitively to the parts of organisms and to the lineages of which organisms are parts.
}

semaphoront (e.g. serial homologs, antimeric homologs, duplicated genes), parts of non-equivalent semaphoronts (e.g. sexual homologs, the adult sphenethmoid and larval chondrocranium), and logically dependent transformation series (e.g. 1. gain or loss of limbs [present/absent]; 2. transformation of limb color [red/blue/green]; 3 . transformation of limb length [short/medium/long]; 4. transformation of number of limbs [two/four]). In each case, independent evolutionary transformation events determine historical identity, i.e. homology.

Synapomorphy, in contrast, refers to the shared occurrence of a derived (apomorphic) character-state, whether or not that shared occurrence resulted from the same transformation event (homology) or different transformation events. In developing his concept of "taxic homology", Patterson (1982b; see also de Pinna, $1991)^{4}$ equated synapomorphy and homology, a move that logically precludes homology as an explanans of synapomorphy. If "homology" = "synapomorphy", and "synapomorphy" = "shared, derived characterstate", then, transitively, "shared derived characterstate due to homology" = "homology due to homology", a tautologous and therefore empty statement. This does not mean that phylogenetic inference is necessarily circular; however, it does mean that a new word would have to be coined to designate the explanans of synapomorphy. Of course, the meaning attached to words is free to change, but we see nothing to be gained by this particular semantic shift, and the linguistic imprecision it engenders seems reason enough to stick with the words and meanings biologists are used to.

A further consequence of conceptualizing characters as transformation series is that de Pinna's (1991) distinction between "primary" and "secondary" homology dissolves. de Pinna (1991, p. 372) believed that homology assessment necessarily requires separate procedures and criteria for hypothesis "generation and legitimation", the former rooted in notions of similarity and the latter based on the simultaneous test of character congruence. However, all possible hypotheses of homology are defined a priori by pure logic alone as a function of the number of heritable parts identified for each terminal (cf. Wheeler, 1998), just as all possible hypotheses of phylogeny are pre-defined as a function of

\footnotetext{
${ }^{4}$ Patterson's arguments for equating homology with synapomorphy devolve to the familiar thesis of pattern cladism. As he stated elsewhere (Patterson, 1982a, p. 305; our italics), "[e]very homology characterizes a group at some level in the hierarchy, and symplesiomorphy and synapomorphy are terms for homologies that stand in hierarchic relation: a symplesiomorphy (general character) makes a group, and a synapomorphy (special character) makes a subgroup. In this light, too, the rift between [pattern] cladists and [phylogenetic] systematists comes clearer into focus." As he went on to conclude (p. 306), "if we are taught, as we have been, to see that pattern through the spectacles of evolutionary theory, how could the pattern ever test the theory?"
} 
the number of terminals (Siddall and Kluge, 1997). As such, no special procedure is required to generate hypotheses of homology; they already exist. Moreover, as reviewed by Grant and Kluge (2003), the origin of hypotheses has no bearing on their scientific status or objective support.

Conceptually, homology refers only to the historical identity of parts related through a series of evolutionary transformation events, and to conclude that the refutation of a hypothesis of homology by the test of character congruence "does not invalidate a primary homology proposition" (de Pinna, 1991, p. 373) conflates the concept of homology with the discovery operations employed to detect instances of it (Grant, 2002) and illustrates the difficulties that arise from treating synapomorphy and homology as synonyms. Synapomorphies that optimize to different nodes of a most parsimonious cladogram resulted from different transformation events and are therefore non-homologous at that level of generality (Farris, 1970), their homology as independent parts of a more inclusive historical individual being beside the point.

The "duality" that de Pinna (1991, p. 372) aimed to accommodate is not due to different concepts or to hypothesis "generation and legitimation", but rather to the fact that the discovery operations employed to test homology claims and detect historical identity are attributable to two classes, one involving separate tests of each transformation series in isolation, the other involving the combined or multiple test of character congruence. These two classes of discovery operations are complementary (Grant, 2002) and are typically employed sequentially in research cycles, a procedure that maximizes severity of test and leads to the progressive elimination of error through reciprocal illumination (Kluge, 1998, 2003b). However, the combination of both kinds of discovery operations is not necessary to legitimately test hypotheses of homology. For some kinds of evidence (e.g. most phenotypic data), structural and/or developmental complexity may provide evidence to defensibly choose among competing hypotheses of homology; those hypotheses that are refuted in this initial round of testing are never submitted to the simultaneous test of congruence. For other kinds of evidence, parts may be so simplified as to be physically indistinguishable, regardless of their historical identities (e.g. nucleotides in DNA sequences), or what evidence that exists may be inadequate to decisively select one hypothesis over another. Separate tests of homology are inert in such cases, and only the test of congruence can lead to an objectively defensible choice among the competing hypotheses (which is the rationale behind methods of direct optimization; e.g. Wheeler, 1996). This decoupling of the two classes of discovery operations invalidates the claim that hypotheses of homology not subjected to the test of congru- ence are not phylogenetic (Rieppel, 1988; de Pinna, 1991) and obviates the requirement that assessment of "primary homology" necessarily precede assessment of "secondary homology" (de Pinna, 1991). We caution that this decoupling is not meant as an endorsement of or justification for decreased testing; both classes of tests should be applied whenever possible, as this procedure maximizes severity of test (Kluge, 1998, 2003a,b).

\section{The limited relevance of properties}

As mentioned above, characters cannot be conceptualized legitimately as properties, because properties are abstract, spatio-temporally unrestricted, and immutable, and are generally conceived as playing a role in classification, whereby possession of a specified property(ies) is both necessary and sufficient for membership in a class (Kluge, 2003b). This suggests that properties can play no role whatsoever in phylogenetic systematics, given that it is concerned with concrete, spatio-temporally restricted, transformable individuals that are related by virtue of being part of a lineage system (Lidén, 1990). Indeed, character individuation in phylogenetics is usually done entirely ostensively, i.e. through a direct comparison of specimens, photographs, drawings, recordings, or other renderings, with naming and description of properties only being used to communicate findings. Nevertheless, in addition to the social value of properties in communication, properties may also be of some cognitive value as descriptors of variation, if those descriptors are used to delimit test statements/conditions in the inference of historical identity/transformation. Arguably, tests may run the risk of being ad hoc and their results meaningless unless relevant properties are specified a priori (i.e. aspects of things conjectured to have undergone heritable transformation). In this context, properties may facilitate generalization across particulars, i.e. they may be useful in defining general test statements. Given that the real criterion of historical identity - being derived from the same transformation event - cannot be determined directly, systematists may infer historical identity indirectly on the basis of observable variation (Hennig, 1966, pp. 93-94; Farris, 1967). Although the very concept of transformation implies properties (i.e. transformation of some thing into another implies a change in some property), a single heritable transformation may entail any number of properties (cf. Hennig, 1966, pp. 92-93), because the number of properties of both the original and transformed things is infinite. What is ultimately proposed as a historical identity relation-a statement of homology-is, however, a consequence of both tests of character analysis/reanalysis and character congruence (Kluge, 2003b). 
The relevance of properties is therefore as delimiters of test statements in the inference of transformation, and not as explanatory elements unto themselves. Used judiciously, they can help to bridge the gap between observation and inference, and facilitate hypothesis testing. On the other hand, concepts that identify similarity as the definiens of character (e.g. Rieppel and Kearney, 2002) commit category errors, treating properties as if they were explanans rather than mere descriptors and conflating intensionally defined class concepts with ostensively defined individuals. Any real thing or event has an infinite number of properties (Grant, 2002; Kluge, 2003b), and care must be taken not to over-interpret or reify these abstract class concepts (Frost and Kluge, 1994; Kluge, 2003b). All that matters in the science of phylogenetic systematics is the inference of heritable transformation, and properties are not necessary to achieve that end.

\section{Discussion}

In two recent papers on character individuation and phylogenetic inference, Richards concluded that "no character individuation principle identified so far is adequate" (Richards, 2003, p. 264) and, further, that "[t]he failure to formulate a criterion of character individuation, raises concerns that character individuation, and therefore the application of parsimony, is either arbitrary or subjective" (Richards, 2002, p. 17). Insofar as his assessment did not include Hennig's (1966) concept of character as transformation series, we are not surprised by his conclusion. Nor are we surprised by his conclusion that phenetic approaches that rely on "mere" observation are unpromising, and that "[a] satisfactory character individuation scheme-and character concept-must surely function to support [the] goal... of the reconstruction of the branching order of the evolutionary past" (Richards, 2003, p. 278). Had Richards considered Hennig's transformationist views and repeated arguments against similarity, he may have been surprised to find that considerable progress had already been achieved in this "project worthy of pursuit" (p. 278).

Likewise, although we do not deny the role played by so-called Kuhnian values in the scientific endeavor (see Grant and Kluge, 2003), this does not mean that character choice and individuation are necessarily arbitrary or subjective and, therefore "the application of parsimony depends on persuasion, background, training and tradition" (Richards, 2002, p. 1). Certainly, the choice to behave rationally is personal (and therefore subjective), and significant social payoffs may be obtained from discarding consistency or other cognitive values, but these observations do nothing to undermine the claim that the application of phylogenetic parsimony in a total evidence analysis of equally weighted evidence minimizes the total number of hypotheses of transformation required to explain the heritable variation observed among species and, as such, the most parsimonious cladogram represents the objectively optimal phylogenetic theory. Furthermore, the fact that there is the potential for error in the delimitation of transformation series does not mean that character individuation is necessarily subjective or arbitrary. Like any scientific hypothesis, proposed character-states and the transformation series through which they are related are open to refutation and refinement through cycles of empirical testing and re-testing (Kluge, 2003b).

Practically minded workers are often tempted to dismiss ontological discussions as purely academic with no relevance to the day-to-day pursuits of practicing scientists, preferring instead to adopt an operationalist perspective. For example, as Brower and Schawaroch (1996, p. 266; see also Brower, 2000) stated:

\footnotetext{
"We are not especially concerned with an explicit ontological definition for homology. Equating homology with synapomorphy only foists its metaphysical baggage onto the latter concept. However, we will claim that, since evolution has nothing to do with the successful discovery and interpretation of characters, evolutionary definitions are clearly superfluous. Practically speaking, homology may not require an operational definition more complicated than similarity, as ordered by the cladistic method'."
}

However, because scored characters provide the basis for phylogenetic analysis, the way character is defined conceptually (ontologically) has a profound effect on all aspects of phylogenetic inference. As practicing systematists are all too aware, the empirical individuation of each transformation series may engender its own unique set of problems, and there is no limit to the kinds of discovery operations and number of tests that can be brought to bear on a particular hypothesis of historical identity. Although the present paper is not primarily concerned with the operational aspects of character individuation, it is clear that the transformation series concept of character allows discovery operations and tests to be assessed for their consistency. Above we discussed briefly the impact of this concept of character on the treatment of polymorphism, different kinds of non-independence, and understanding of homology, but its effects can also be seen in approaches to multiple sequence alignment, character weighting, statistical methods of phylogenetic analysis, and beyond.

The science of phylogenetic systematics has progressed impressively beyond Hennig's (1966) original formulation, both conceptually and operationally. Nevertheless, Hennig's arguments played an important role in establishing the analytical framework for modern systematics, and they represent a key starting point in the elimination of pattern cladistics and the "point-andclick" approach to systematics that has taken hold in recent years (Grant et al., 2003). 


\section{Acknowledgments}

We gratefully acknowledge the critical suggestions from Richard Richards and two anonymous reviewers. Our ideas also benefited greatly from discussions with Jan De Laet, Darrel Frost, Julián Faivovich, Mike Novacek, Leo Smith, John Wenzel, and Ward Wheeler, although their inclusion here should not be taken as an endorsement of our views, and all errors are our own. Support for T.G. was provided by an AMNH Graduate Student Fellowship, a Columbia University/CERC Faculty Fellowship, and NSF DEB 0309226, and for A.G.K. by the Cladistics Institute, Harbor Springs, MI.

\section{References}

Agosti, D., Jacobs, D., DeSalle, R., 1996. On combining protein sequences and nucleic acid sequences in phylogenetic analysis: The homeobox protein case. Cladistics, 12, 65-82.

Bodenbender, B.E., Fisher, D.C., 2001. Stratocladistic analysis of blastoid phylogeny. J. Paleont. 75, 351-369.

Boyd, R., 1999. Homeostasis, species, and higher taxa. In: Wilson, R.A. (Ed.), Species: New Interdisciplinary Essays. MIT Press, Cambridge, MA, pp. 141-185.

Brochu, C.A., Bryant, H.N., Theodor, J.M., O’Leary, M.A., Adrain, J.M., Sumrall, C.D., 2001. Modern phylogenetics in paleontology: comments on Vermeij 1999. Paleobiology, 27, 174-176.

Brower, A.V.Z., 2000. Evolution is not a necessary assumption of cladistics. Cladistics, 16, 143-154.

Brower, A.V.Z., Schawaroch, V., 1996. Three steps of homology assessment. Cladistics, 12, 265-272.

Colless, D.H., 1985. On 'character' and related terms. Syst. Zool. 34, 229-233.

Darwin, C., 1859 On the Origin of Species by means of Natural Selection, or the Preservation of Favoured Races in the Struggle for Life. John Murray, London. [1964. Facsimile Reprint of the 1st edn. Harvard University Press, Cambridge, MA.]

Eldredge, N., 1979. Alternative approaches to evolutionary theory. Bull. Carnegie Mus. Nat. Hist. 13, 7-19.

Faivovich, J., 2002. On RASA. Cladistics, 18, 324-333.

Farris, J.S., 1967. The meaning of relationship and taxonomic procedure. Syst. Zool. 16, 44-51.

Farris, J.S., 1970. Methods for computing Wagner Trees. Syst. Zool. 19, 83-92.

Farris, J.S., 1983. The logical basis of phylogenetic analysis. In: Platnick, N.I., Funk, V.A. (Eds.), Advances in Cladistics. Columbia University Press, New York, pp. 7-36.

Farris, J.S., 2000. Paraphyly, outgroups, and transformations. Cladistics, 16, 425-429.

Farris, J.S., 2002. RASA attributes highly significant structure to randomized data. Cladistics, 18, 334-353.

Farris, J.S., Kluge, A.G., De Laet, J., 2001. Taxic revisions. Cladistics, 17, 79-103.

Fisher, D.C., 1994. Stratocladistics: morphological and temporal patterns and their relation to phylogenetic process. In: Grande, L., Rieppel, L. (Eds.), Interpreting the Hierarchy of Nature: from Systematic Patterns to Evolutionary Process Theories. Academic Press, San Diego, pp. 133-171.

Fisher, D.C., Bodenbender, B.E., 2003. Blastoid stratocladisticsreply to Sumrall and Brochu. J. Paleont. 77, 195-198.

Fox, D.L., Fisher, D.C., Leighton, L.R., 1999. Reconstructing phylogeny with and without temporal data. Science, 284, 18161819.
Freudenstein, J.V., Pickett, K.M., Simmons, M.P., Wenzel, J.W., 2003. From basepairs to birdsongs: phylogenetic data in the age of genomics. Cladistics, 19, 333-347.

Fristrup, K., 1992. Character: current usages. In: Keller, E.F. Lloyd, E.A. (Eds.), Keywords in Evolutionary Biology. Harvard University Press, Cambridge, MA, pp. 45-51.

Frost, D.R., Kluge, A.G., 1994. A consideration of epistemology in systematic biology, with special reference to species. Cladistics, 10 , 259-294.

Geiger, D.L., Fitzhugh, K., Thacker, C.E., 2001. Timeless characters: a response to Vermeij (1999). Paleobiology, 27, 177-178.

Ghiselin, M.T., 1974. A radical solution to the species problem. Syst. Zool. 23, 536-544.

Grant, T., 2002. Testing methods: The evaluation of discovery operations in evolutionary biology. Cladistics, 18, 94-111.

Grant, T., Faivovich, J., Pol, D., 2003. The perils of 'point-and-click' systematics. Cladistics, 19, 276-285.

Grant, T., Kluge, A.G., 2003. Data exploration in phylogenetic inference: Scientific, heuristic, or neither. Cladistics, 19, 379-418.

Hennig, W., 1966. Phylogenetic Systematics. University of Illinois Press, Chicago, IL.

Heyning, J.E., Thayer, M.K., Fisher, D.C., Fox, D.L., Leighton, L.R., 1999. Phylogenies, temporal data, and negative evidence. Science, 285, 1179a.

Hull, D.L., 1988. Science as a Process: An Evolutionary Account of the Social and Conceptual Development of Science. University of Chicago Press, Chicago, IL.

Kluge, A.G., 1990. Species as historical individuals. Biol. Philos. 5, 417-431.

Kluge, A.G., 1998. Sophisticated falsification and research cycles: Consequences for differential character weighting in phylogenetic analysis. Zool. Scripta, 26, 349-360.

Kluge, A.G., 2003a. On the deduction of species relationships: a précis. Cladistics, 19, 233-239.

Kluge, A.G., 2003b. The repugnant and the mature: Atemporal similarity and historical identity in phylogenetic inference. Cladistics, 19, 356-368.

Kluge, A.G., Farris, J.S., 1999. Taxic homology = overall similarity. Cladistics, 15, 205-212.

Lidén, M., 1990. Replicators, hierarchy, and the species problem. Cladistics, 6, 183-186.

Novacek, M.J., 2001. Character distributions are phylogenetic evidence. Paleobiology, 27, 172-173.

Patterson, C., 1982a. Cladistics and classification. New Scientist, 94, 303-306.

Patterson, C., 1982b. Morphological characters and homology. In: Joysey, K.A., Friday, A.E. (Eds.), Systematics Association Special Volume 21: Problems of Phylogenetic Reconstruction. Academic Press, New York, pp. 21-74.

de Pinna, M.C.C., 1991. Concepts and tests of homology in the cladistic paradigm. Cladistics, 7, 367-394.

Platnick, N.I., 1979. Philosophy and the transformation of cladistics. Syst. Zool. 28, 537-546.

Richards, R., 2002. Kuhnian values and cladistic parsimony. Perspect. Sci. 10, 1-27.

Richards, R., 2003. Character individuation in phylogenetic inference. Phil. Sci. 70, 264-279.

Rieppel, O., 1988. Fundamentals of Comparative Biology. Birkäuser, Basel, Switzerland.

Rieppel, O., Kearney, M., 2002. Similarity. Biol. J. Linn. Soc. 75, 5982.

Siddall, M.E., Kluge, A.G., 1997. Probabilism and phylogenetic inference. Cladistics, 13, 313-336.

Smith, A.B., 2000. Stratigraphy in phylogeny reconstruction. J. Paleont. 74, 763-766.

Stamos, D.N., 2001. Quantum indeterminism and evolutionary biology. Phil. Sci. 68, 164-184. 
Sumrall, C.D., Brochu, C.A., 2003. Resolution, sampling, higher taxa and assumptions in stratocladistic analysis. J. Paleont. 77, 189-194.

Vermeij, G.J., 1999. A serious matter with character-taxon matrices. Paleobiology, 25, 431-433.

Vermeij, G.J., 2001. Time and the comparative method. Paleobiology, 27, 179-180.

Wagner, G.P. (Ed.), 2001. The Character Concept in Evolutionary Biology. Academic Press, San Diego, CA.

Wheeler, W.C., 1996. Optimization alignment: the end of multiple sequence alignment in phylogenetics? Cladistics, 12, 1-9.
Wheeler, W.C., 1998. Alignment characters, dynamic programming and heuristic solutions. In: DeSalle, R., Schierwater, B. (Eds.), Molecular Approaches to Ecology and Evolution, 2nd edn. Birkhäuser, Basel, Switzerland, pp. 243-251.

Wilson, D.S., Sober, E., 1994. Reintroducing group selection to the human behavioral sciences. Behav. Brain Sci. 17, 585-654.

Woodger, J.H., 1929. Biological Principles: A Critical Study. Harcourt, Brace \& Co., New York.

Woodger, J.H., 1952. From biology to mathematics. Br. J. Phil. Sci. 3, $1-21$ 